\title{
Validation of the glasgow antipsychotic side-effect scale (gass) in greece
}

\begin{abstract}
The aim of the present study was to evaluate the linguistic adaptation and psychometric validation into the Greek language of the GASS scale for the assessment of side effects in patients treated with second generation antipsychotic medication. The GASS scale takes 5 minutes to complete ( 21 items for men and women) and contains self-explanatory questions in everyday plain English while providing a structured systematic method of reviewing antipsychotic side effects. The translation and cultural adaptation of the questionnaire was performed according to international standards. Internal consistency using the Cronbach $\alpha$ coefficient and test-retest reliability using the intraclass correlation coefficient (ICC) was used to assess the reliability of the instrument. Patient's sample consisted of 80 participants with a mean age of 42.6years. Internal consistency and intraclass correlation coefficient were adequate (Cronbach $\alpha=0.79$ and $\mathrm{ICC}=0.96)$. The test-retest percent agreement for the aforementioned categories was 95.9. Agreement was satisfactory according to Kappa coefficient which was equal to $0.78(\mathrm{p}<0.001)$. The Greek validation of the GASS scale shows appropriate feasibility, reliability, and discriminative performance as a patientreported outcome to be used for the assessment of the impact of side effects on patients with schizophrenia.
\end{abstract}

Volume I Issue 4 - 2014

\author{
Nystazaki Maria,' Tsapakis Eva Maria, ${ }^{2}$ \\ Hadjulis Michael,' Alevizopoulos Giorgos' \\ 'Department of Psychiatry, University of Athens, Greece \\ ${ }^{2}$ Aghios Charalambos Mental Health Clinic, Greece
}

Correspondence: Nystazaki Maria, Department of Psychiatry, Agioi Anargyroi Hospital,Timiou Stavrou and Noufaron, 14564 Kalyftaki, Kifissia, Athens, Greece, Tel 302000000000 , Email nystazaki@yahoo.gr

Received: July 30, 2014 | Published: August 04, 2014

Keywords: second generation antipsychotics, gass scale, validation

Abbreviations: SGAs, second generation antipsychotics; FGA, first-generation antipsychotics; GASS, glasgow antipsychotic side-effect scale; DDDs, defined daily doses; LUNSERS, liverpool university neuroleptic side effect rating scale; UKUSERS, udvalg for kliniske undersogelser side-effect rating scale; SMARTS, systematic monitoring of adverse events related to treatments; ANNSERS, nonneurological side effect rating scale

\section{Introduction}

Schizophrenia is a chronic mental illness that requires long-term antipsychotic treatment to both manage disease symptoms and delay relapses. ${ }^{1}$ The discovery of antipsychotic medications has improved outcomes in persons with schizophrenia through control of symptoms. ${ }^{2}$ Newer antipsychotic treatments have proven useful in reducing both relapses and the severity of symptoms. ${ }^{3}$

The past years the prescription of SGAs has increased. ${ }^{4}$ The Service Division of Scotland reported that all NHS Boards except NHS Shetland show increased prescribing of antipsychotic drugs since 2008/2009. The use of antipsychotic drugs has increased from 7.0 to 8.0 DDDs per 1,000 populations per day between 2008/2009 and $2012 / 2013$. From 2006 to 2011 there has been a dramatic 59\% increase in the use of SGAs medication. ${ }^{5}$ In Greece the last 5years there has been a documented $18.6 \%$ increase in the use of antipsychotic drugs. ${ }^{6}$

Second generation antipsychotics (SGAs) are generally preferred over typical antipsychotics for schizophrenia treatment as they are associated with fewer extra pyramidal symptoms, lower risk of tardive dyskinesia, and possibly greater improvement in negative symptoms. ${ }^{7}$ Schizophrenia and bipolar disorder are examples of chronic illnesses a high risk of relapse associated with major functional consequences. ${ }^{8}$ One of the contributing factors to relapse in schizophrenia is poor or partial adherence to medication. ${ }^{9}$ Use of the second-generation antipsychotics, which have a different adverse event profile than the first-generation antipsychotics, was hoped to improve adherence ${ }^{10}$ and, consequently, treatment outcomes compared with firstgeneration antipsychotics ${ }^{11,12}$ However, treatment adherence remains low. ${ }^{11}$ Nonadherence significantly increases the risk of relapse and is associated with impaired functional outcomes in schizophrenia. ${ }^{13,14}$ In a systematic review Olivares $\mathrm{J}^{15}$ found that non- adherence to antipsychotic medication was the most frequent reported factor that may drive to relapse, he also reported that treatment related factors such as side-effects were associated with increased relapse rates. ${ }^{15}$

The majority of patients with schizophrenia (84\%) discontinue their index antipsychotic during the follow-up period and in the longterm 40 to $50 \%$ seem to be non-compliant with no real difference in terms of adherence between first-generation antipsychotics (FGA) and second-generation antipsychotics (SGA). ${ }^{16,17}$ The most striking result of the CATIE study, which enrolled almost 1,500 individuals with chronic schizophrenia, was the high rate of treatment discontinuation (up to $74 \%$ ) over the 18-month period of the trial and the short median time to discontinuation of treatment (about 6 months) in all phases of the trial. ${ }^{18}$

Antipsychotic drugs potentially cause a wide range of adverse effects including extra pyramidal symptoms, sedation, weight gain, metabolic disturbance, sexual dysfunction, urinary symptoms, gastrointestinal symptoms, and symptoms that reflect raised prolactin, for example, menstrual irregularities and galactorrhoea. Adverse effects are clinically important as they add to the suffering of patients, impair quality of life, can be stigmatizing and often lead to poor or lack of adherence with antipsychotic medication, which in turn usually leads to relapse of the underlying psychiatric disorder. ${ }^{19}$

Guidelines recommend that side effects should be monitored regularly. ${ }^{20-23}$ Many efforts have been made to develop or validate specific instruments to assess side effects of medication used in schizophrenia. Antipsychotic side effect rating scales were first introduced in the 1970s. Since then a number of side effect and adverse effect scales have been developed..$^{24,25}$ 
Each and every developed side effect scale, currently in use, has its own specific features. Some assess only specific side effects ${ }^{24}$ whereas some assess a range of side effects..$^{25-29}$ Some are completed by clinicians, whereas others can be completed by patients. ${ }^{25,26,29,30}$

A number of scales focus primary on EPS such as the Abnormal Involuntary Movement Scale (AIMS), ${ }^{28}$ extra pyramidal Symptom Rating Scale (ESRS), ${ }^{25}$ the Simpson-Angus Scale (SAS) ${ }^{24}$ and the Udvalg for Kliniske Undersogelser Side-Effect Rating Scale (UKUSERS). ${ }^{26}$

Newer side effect rating scales have been developed to asses a range of side effects such as the Antipsychotic Non-Neurological Side Effect Rating Scale (ANNSERS), ${ }^{27}$ a physician- or self- rated scale addressing a wide range of FGA and SGA side effects.

The Liverpool University Neuroleptic Side-Effect Rating Scale (LUNSERS) ${ }^{29}$ is one of the most popular self-completed side effect rating scales in Britain assessing a wide range of side effects includes 41 items, plus 10 'red herring' items but is time consuming and one word symptoms can be difficult for patients to understand.

Other newer self-completed side effect scales include the Systematic Monitoring of Adverse events Related to Treatments (SMARTS) $^{30}$ and the Glasgow Antipsychotic Side-effect Scale (GASS). ${ }^{25}$

The SMARTS checklist assesses whether patients are currently 'troubled' by 11 well-established potential antipsychotic side effects. Patients provide their responses to these questions by circling relevant side effects. An additional open question enquires about any other possible side effects. ${ }^{31}$

Among currently used scales, the GASS is one of the most practical for clinical use. It is a self-report rating scale assessing SGA side effects includes ratings of both neuromuscular and metabolic side effects. The scale was calibrated against the Liverpool University Neuroleptic Side-Effect Rating Scale (LUNSERS) ${ }^{29}$ in 50 outpatients on SGAs, compared to 50 healthy individuals not on antipsychotics, and was found to have good discriminatory power; construct validity; and test-retest reliability.

The GASS takes 5minutes to complete (21 items for men and women) and contains self-explanatory questions in everyday plain English while providing a structured systematic method of reviewing antipsychotic side effects. ${ }^{31}$

The aim of the present study was to evaluate the linguistic adaptation and psychometric validation into the Greek language of the GASS for the assessment of side effects in patients treated with second generation antipsychotic medication.

\section{Materials and methods}

\section{Participants}

The study was carried out at 2 Psychiatric Clinics in Greece. In particular at the Depot and Clozapine Clinic of Agioi Anargyroi Hospital Department of Psychiatry and at the Depot of "Aghios Charalambos" Mental Health Clinic. Inclusion criteria included participants' age being at least 18 years, having a diagnosis of schizophrenia or schizoaffective disorder as established by the Structured Clinical Interview for DSM-IV, being adherent to treatment and currently prescribed and taking SGA (regardless of other concomitant medication). All participants were above the 8 grade level status.
After complete description of the study to the participants, written informed consent was obtained. Permission to conduct the Validation of the GASS Scale was obtained by the author of the original instrument. Ethical approval for the study was granted by the Research and Ethics Committee of Agioi Anargyroi Hospital.

\section{Translating the scale}

The GASS scale was translated into Greek from the original questionnaire as recommended in the literature review. ${ }^{32}$ Upon agreement, two professional translators, native speakers of the Greek (i.e. target) language and fluent in the English (i.e. source) language undertook independent forward translations into the target language. A reconciled version of the instrument was developed and a backward translation of this reconciled version back into the original language was performed by a professional translator. The back-translation and the original one were compared and any discrepancies between them led to changes in the reconciled translation in the Greek language. An expert committee reviewed this latest version and gave their feedback. At the next stage, the questionnaire was administered to a small group of patients who volunteered to take part at the cognitive debriefing phase in order to assess clarity and comprehension of the questionnaire items. After this final feedback, the final Greek version was produced.

\section{Statistical analysis}

Internal consistency and reliability was determined by calculation of the Cronbach $\alpha$ coefficient. Cronbach $\alpha$ equal to or greater than 0.70 was considered acceptable. ${ }^{33}$ Student's t-tests were used to compare mean values. To conceptualize the overlap, between patient and controls, effect size was also reported. According to the literature, effect sizes of 0.2-0.5 are considered small, between 0.51 and 0.80 moderate and over 0.8 effect- sizes are considered large. ${ }^{34}$ To assess the consistency of the scores, test-retest stability was determined by administering the GASS questionnaire to 80 patients on two separate occasions one week apart. Intra-class correlation coefficients (ICCs) were computed to determine the level of agreement. ICCs equal or lower to 0.40 indicate poor to fair agreement, $0.41-0.60$ moderate agreement, $0.61-0.80$ good agreement and over 0.80 excellent agreements. ${ }^{35}$ Paired $\mathrm{t}$-tests were also used to investigate the differences after one week re-administration. Kappa values were calculated to assess the agreement for the categorization of patients into three groups (Absent/mild, moderate and severe side effects) between first and second administration. The maximum Kappa value is 1 , corresponding to perfect agreement, values greater or equal to 0.75 are considered as excellent agreement and values greater than 0.4 indicate acceptable reliability. ${ }^{36} \mathrm{P}$ values reported are two-tailed. Statistical significance level was set at .05 and analysis was conducted using SPSS 19.0.

\section{Results and discussion}

Patient's sample consisted of 80 participants $(68.8 \%$ men and $31.3 \%$ women) with a mean age of 42.6 years ( $\mathrm{SD}=14.5$ years). All patients were on second generation antipsychotic medication either on long acting formulations or clozapine, as follows: 5 patients were on clozapine, 40 olanzapine long acting, 25 risperidone long acting and 10 paliperidone long acting respectively. All dosing regimens were within BNF limits. Approximately a third of the patients, 31.3\%, were on monotherapy, whereas the remaining $68.7 \%$ was on combination with antidepressants $(11.3 \%)$ or mood stabilizers $(67.5 \%)$. The control group consisted of 50 participants ( $70 \%$ men and $30 \%$ women) with a mean age of 43.4 years ( $\mathrm{SD}=13.9$ years). The sample's characteristics are shown in (Table 1). 
Table I Samples characteristics

\begin{tabular}{llll}
\hline & $\begin{array}{l}\text { Patients } \\
\text { N (\%) }\end{array}$ & $\begin{array}{l}\text { Controls } \\
\text { N (\%) }\end{array}$ & P \\
\hline $\begin{array}{l}\text { Age(years), mean (SD) } \\
\text { Sex }\end{array}$ & $42.6(14.5)$ & $43.4(\mid 3.9)$ & $0.756^{*}$ \\
Men & $55(68.8)$ & $35(70.0)$ & $0.88 I^{* *}$ \\
Women & $25(31.3)$ & $15(30.0)$ & \\
SGA & & & \\
No & $0(0.0)$ & & \\
Yes & $80(100)$ & & \\
Antidepressants & & & \\
No & $71(88.8)$ & \\
Yes & $9(11.3)$ & & \\
Polypharmacy & & \\
No & $71(88.8)$ & \\
Yes & $9(11.3)$ & \\
Moodstabilizer & & \\
No & $26(32.5)$ & \\
Yes & $54(67.5)$ & \\
Monotherapy & & \\
No & $55(68.8)$ & \\
Yes & $25(31.3)$ & \\
\hline
\end{tabular}

*Student's t-test; $* *$ chi-square test

Second generation antipsychotics (SGAs)

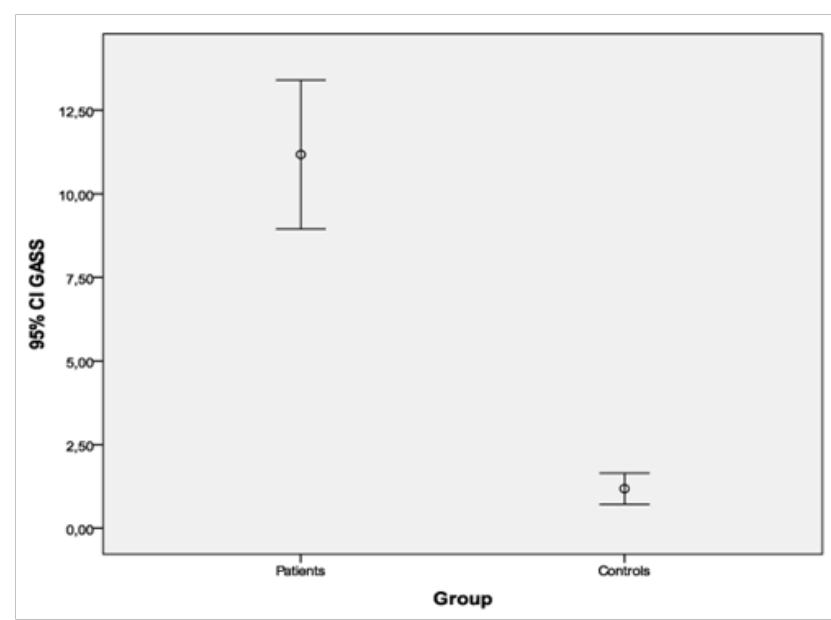

Figure I Mean GASS values for patient and control groups.

Cronbach's $\alpha$ for the GASS scale was 0.79 and exceeded the minimum reliability standard of 0.70 indicating acceptable internal consistency reliability. ${ }^{33}$ Mean scores of the GASS scale was 11.2 $(\mathrm{SD}=10.0)$ for the patient's group and $1.2(\mathrm{SD}=1.6)$ for the control group (Figure 1). The difference between patients and controls was statistically significant $(\mathrm{p}<0.001)$ with a large effect size equal to 1.39 showing the discriminative validity of the questionnaire. ${ }^{34} 10 \%$ of patients were categorized as having moderate side effects and $90 \%$ were categorized as having absent/mild side effects. All controls were categorized as having absent or mild side effects. After excluding patients on polypharmacy, the difference between patients and controls still remained statistically significant $(\mathrm{p}<0.001)$.

Test retest stability analysis showed excellent agreement with ICC equal to 0.96 (95\% CI: 0.93-0.98, $\mathrm{p}<0.001)$. The mean GASS was $9.9(\mathrm{SD}=7.9)$ at the first administration and $10.1(\mathrm{SD}=8.1)$ at the second administration after one week from the first $(p=0.608)$. Subjects at the first and second administration were categorized in three groups (Absent/mild, moderate and severe side effects). The test-retest percent agreement for the aforementioned categories was 95.9. Agreement was satisfactory according to Kappa coefficient which was equal to $0.78(\mathrm{p}<0.001)$.

GASS scores according to patient's characteristics are shown in (Table 2). There were no statistically significant difference amongst monotherapy and polypharmacy. The concomitant use of antipsychotics and mood stabilizers had no significant effect on GASS scores. However, women and patients receiving antidepressants reported statistically significant higher scores in GASS, $\mathrm{p} \leq 0.008$ and $p \leq 0.038$ respectively. Our findings are similar to other studies. Women are more susceptible to side effects due to antipsychotic medication. Women's bodies, on average, contain $25 \%$ more adipose tissue than those of men. ${ }^{37}$ Since most antipsychotic drugs are highly lipophilic molecules they might be accumulated in lipid stores resulting the development of noticeable side effects. ${ }^{37}$ Furthermore the use of antidepressants in patients who suffer from schizophrenia might represent a progressive stage of the illness with chronicity and negative symptoms, inadequate response to the antipsychotic medication, or failure of multiple challenges with antipsychotic medications with accumulated side effects. The use of antidepressants in patients suffering from schizophrenia is rather controversial, except the decreased risk for suicidal behaviour. ${ }^{38}$

2 Mean GASS scores according to patient's characteristics

\begin{tabular}{|c|c|c|c|}
\hline & GASS & & $\mathbf{P}$ \\
\hline & Mean & SD & \\
\hline \multicolumn{4}{|l|}{ Sex } \\
\hline Men & 9.8 & 9.6 & 0.008 \\
\hline Women & 14.2 & 10.3 & \\
\hline \multicolumn{4}{|c|}{ Antidepressants } \\
\hline No & 10.3 & 9.5 & 0.038 \\
\hline Yes & 17.7 & 12.1 & \\
\hline \multicolumn{4}{|c|}{ Polypharmacy } \\
\hline No & $1 \mathrm{I} .44$ & 10.5 & 0.495 \\
\hline Yes & 9 & 5.1 & \\
\hline \multicolumn{4}{|c|}{ Moodstabilizer } \\
\hline No & 13.2 & 9.9 & 0.219 \\
\hline Yes & 10.2 & 9.9 & \\
\hline \multicolumn{4}{|c|}{ Monotherapy } \\
\hline No & 10.2 & 9.9 & 0.179 \\
\hline Yes & 13.4 & 10.1 & \\
\hline
\end{tabular}

\section{Glasgow Antipsychotic Rating Scale (GASS)}

The GASS scale was developed by Waddell $\mathrm{L}$ and Taylor $\mathrm{M}$ in 2008 and is currently amongst the most popular and easy to use antipsychotic side- effect rating scales in the UK. ${ }^{31,39}$ The scale has not been previously translated or validated in Greek population. It is the first time a self rating scale related to adverse events of antipsychotic drugs is being validated in the Greek language. Our results show that the GASS scale presents adequate characteristics for use in patients with schizophrenia and schizoaffective disorder in Greece. The Cronbach's alpha value for the scale was 0.79 ; indicating acceptable internal consistency reliability and the differences in mean scores of the GASS scale between patients and controls show the discriminative validity of the questionnaire. Regarding reliability, coefficient alpha estimates of the GASS were generally acceptable against the background observations. There are multiple factors for considering a reliability coefficient such as the construct being measured, time available for testing, how the scores are to be used, amongst others, so that reliability coefficients as low as 0.79 are acceptable. Therefore, in 
general the reliability estimates reported here were within acceptable range. GASS might be a simplistic instrument. However, is gathering information that only needs to be used in a practical way rather than inferential way, the reliability and validity requirements are more basic. It is actually measuring the real-world events, such as troubling side-effects, that they are intended to measure. This type of internal validity could be assessed by comparing the questionnaire responses with objective measures of the side effects; for example comparing the self-reported shaking of the hands or arms with some objective measure. Other alternative measures such as Cohen's kappa statistic or the Fleiss kappa, were not performed since they are more complicated and are rather suitable for objective than subjective rating. ${ }^{40,41}$

Since GASS is a simple and easy to administer self-rating scale. The completion time is low $(<10 \mathrm{~min})$ compared with longer questionnaires such as the LUNCERs scale (approx. $20 \mathrm{~min}$ ). ${ }^{25}$ Simple checklists are promoted as more effective clinical intervention for improving service. ${ }^{42}$

\section{Conclusion}

Antipsychotic drugs can cause a wide range of potential adverse effects. ${ }^{19}$ Adverse effects of antipsychotics are often neither diagnosed nor treated ${ }^{43}$ In his audit, Cleary $\mathrm{A}^{43}$ investigated current practice within a rural mental health service area on the monitoring and documentation of side effects of antipsychotic depot medication. According to the audit, side effects were not recorded in $72 \%$ of cases. Locally developed checklists scales were used in $25 \%$ of the case notes examined, this percentage is low when considering the popularity of using an evidenced-based tool in practice, for example, the Liverpool University Neuroleptic Side Effect Rating Scale (LUNSERS) ${ }^{25} \mathrm{He}$ concluded that Collaborative practice with feedback from service users is essential in service improvement and care delivery. ${ }^{43}$

The GASS scale is a valid reliable tool, which could aid systematic clinical assessment. The GASS includes ratings of both neuromuscular and metabolic side effects. It is quick to complete, either for the clinician or patient. The use of a plain language helps comprehension. It is independent of any commercial interest and can be used without cost. $^{31,39}$

Despite the above results, this study presents some limitations that should be considered. The GASS questionnaire is a simple, selfreported screening tool. It is argued to be inadequate to understand some forms of information - i.e. "I felt dizzy when I stood up and/or have fainted" (question n. 3) or "I have been drooling" (question n.8) etc. Furthermore, it is asking only a limited amount of information without explanation and there is no way to tell how truthful a respondent is being. Therefore there is a level of subjectivity that is not acknowledged. The scale was only assessed in outpatients on long acting antipsychotic medication and clozapine, excluding a range of SGAs that do not have a depot formulation and FGAs. The differences in the side-effect profile of the medications, we did not assessed, might influence the screening accuracy of the questionnaire.

Overall the Greek validation of the GASS scale shows appropriate feasibility, reliability, and discriminative performance as a patientreported outcome to be used for the assessment of the impact of side effects on patients with schizophrenia. This information could be very important to improve therapeutic alliances and treatment adherence among patients with schizophrenia. Since drug induced side-effects are pivotal in medication non-adherence for all patients, the current validation of GASS can be a useful study for further assessment of antipsychotic medications in different population samples, such as first episode and bipolar sufferers on antipsychotic drug regimes.

\section{Acknowledgments}

None.

\section{Conflicts of interest}

Author declares there are no conflicts of interest.

\section{Funding}

None.

\section{References}

1. Schultz SH, North SW, Shields CG. Schizophrenia: a review. Am Fam Physician. 2007;75(12):1821-1829.

2. CarpenterWT, Davis JM. Anotherview of the history of antipsychotic drug discovery and development. Mol Psychiatry. 2012;17(12):1168-1173.

3. Buckley PF. Update on the etiology and treatment of schizophrenia and bipolar disorder. CNS Spectr. 2008;13(2 Suppl 1):1-10.

4. Seeman MV. Secondary Effects of Antipsychotics: Women at Greater Risk than Men. Schizophr Bull. 2009;35(5):937-948.

5. NHS Scotland. Prescribing-Medicines used in Mental Health. 2013.

6. National Statistical Service of Greece. 2013

7. Balf G, Stewart TD, Whitehead R, et al. Metabolic adverse events in patients with mental illness treated with antipsychotics: a primary care perspective. Prim Care Companion J Clin Psychiatry. 2008;10(1):15-24.

8. Velligan DI, Weiden PJ, Sajatovic M, et al. Strategies for addressing adherence problems in patients with serious and persistent mental illness: recommendations from the expert consensus guidelines. $J$ Psychiatr Pract. 2010;16(5):306-324.

9. Llorca PM. Partial compliance in schizophrenia and the impact on patient outcomes. Psychiatry Res. 2008;161(2):235-247.

10. Ascher-Svanum H, Faries DE, Zhu B, et al. Medication adherence and long-term functional outcomes in the treatment of schizophrenia in usual care. J Clin Psychiatry. 2006;67(3):453-460.

11. Al-Zakwani IS, Barron JJ, Bullano MF, et al. Analysis of healthcare utilization patterns and adherence in patients receiving typical and atypical antipsychotic medications. Curr Med Res Opin 2003;19(7):619-626.

12. Liu-Seifert H, Osuntokun OO, Feldman PD. Factors associated with adherence to treatment with olanzapine and other atypical antipsychotic medications in patients with schizophrenia. Compr Psychiatry. 2012;53(1):107-115.

13. Gilmer TP, Dolder CR, Lacro JP, et al. Adherence to treatment with antipsychotic medication and health care costs among Medicaid beneficiaries with schizophrenia. Am J Psychiatry. 2004;161(4):692-699.

14. Ascher-Svanum H, Faries DE, Zhu B, et al. Medication adherence and long-term functional outcomes in the treatment of schizophrenia in usual care. J Clin Psychiatry. 2006;67(3):453-460.

15. Olivares JM, Sermon J, Hemels M, et al. Definitions and drivers of relapse in patients with schizophrenia: a systematic literature review. Ann Gen Psychiatry . 2013;12(1): 32.

16. Kreyenbuhl J, Slade EP, Medoff DR, et al. Time to discontinuation of first- and second-generation antipsychotic medications in the treatment of schizophrenia. Schizophr Res. 2011;131(1-3):127-132.

17. Velligan DI, Weiden PJ, Sajatovic M, et al. The expert consensus guideline series: adherence problems in patients with serious and persistent mental illness. J Clin Psychiatry. 2009;70 Suppl 4:1-46. 
18. Lieberman JA, Stroup TS. The NIMH-CATIE Schizophrenia Study: What Did We Learn? Am J Psychiatry. 2011;168(8):770-775.

19. Haddad PM, Sharma SG. Adverse effects of atypical antipsychotics: differential risk and clinical implications. CNS Drugs. 2007;21(11):911936.

20. Lehman AF, Lieberman JA, Dixon LB, et al. Practice guideline for the treatment of patients with schizophrenia. Second edition. Am J Psychiatry. 2004;161(2 Suppl);1-56.

21. Royal Australian and New Zealand College of Psychiatrists clinical practice guidelines for the treatment of schizophrenia and related disorders. Aust N Z J Psychiatry. 2005;39(1-2):1-30.

22. Canadian Psychiatric Association. Clinical Practice Guidelines Treatment of Schizophrenia. Can J Psychiatry. 2005;50(13 Suppl 1):7S-57S.

23. National Institute for Clinical Excellence. Guidance on the Use of Newer (Atypical) Antipsychotic Drugs for the Treatment of Schizophrenia. NICE technology appraisals [TA43]. 2002.

24. Janno S, Holi MM, Tuisku K, et al. Validity of Simpson-Angus Scale (SAS) in a naturalistic schizophrenia population. BMC Neuro. 2005;15(1):5.

25. Chouinard G, Ross-Chouinard A, Annable L, et al. The Extrapyramidal Symptom Rating Scale. Can J Neurol Sci. 1980;7:233.

26. Lingjaerde $\mathrm{O}$, Ahlfors $\mathrm{UG}$, Bech $\mathrm{P}$, et al. The UKU side effect rating scale. A new comprehensive rating scale for psychotropic drugs and a cross-sectional study of side effects in neuroleptic-treated patients. Acta Psychiatr Scand Suppl. 1987; 334:1-100.

27. Yusufi BZ, Mukherjee S, Aitchison $\mathrm{K}$, et al. Inter-rater reliability of the Antipsychotic Non-Neurological Side-Effects Rating Scale (ANNSERS). Schizophr Bull. 2005;31: 574.

28. Rey JM, Hunt GE, Johnson GF. Assessment of tardive dyskinesia in psychiatric outpatients using a standardized rating scale. Aust $N Z J$ Psychiatry. 1981;15(1):33-37.

29. Day JC, Wood G, Dewey M, et al. A self-rating scale for measuring neuroleptic side-effects. Validation in a group of schizophrenic patients. Br J Psychiatry . 1995;166(5):650-653.

30. Peter M Haddad,corresponding author W Wolfgang Fleischhacker Joseph Peuskens, et al. SMARTS (Systematic Monitoring of Adverse events Related to Treatments): The development of a pragmatic patientcompleted checklist to assess antipsychotic drug side effects. Ther Adv Psychopharmacol. 2014;4(1):15-21.
31. Waddell L, Taylor M. A new self-rating scale for detecting atypical or second generation antipsychotic side effects. J Psychopharmacol. 2008;22(3):238-243.

32. Vijver FV, Hambleton RK.Translating tests: Some practical guidelines. European Psychologist. 1996;1:89-99.

33. Bland JM, Altman DG. Cronbach's alpha. BMJ. 1997;314(7080):572.

34. Baugh F. Correcting effect sizes for score reliability: A reminder that measurement and substantive issues are linked inextricably. Edu Psychol Measurement. 2002;62:254-263

35. Shrout PE, Fleiss JL. Intraclass Correlations: Uses in Assessing Rater Reliability. Psychol Bull. 1979;86(2):420-428.

36. Landis JR, Koch GG. The measurement of observer agreement for categorical data. Biometrics. 1977;33(1):159-74.

37. Seeman MV. Gender differences in the prescribing of antipsychotic drugs. Am J Psychiatry. 2004;161(8):1324-1333.

38. Singh SP, Singh V, Kar N, et al. Efficacy of antidepressants in treating the negative symptoms of chronic schizophrenia: meta-analysis. $\mathrm{Br} J$ Psychiatry. 2010;197(3):174-179.

39. Keefe R. Guide to Assessment Scales in Schizophrenia, Springer Healthcare, a part of Springer Sci Business Media. 2012.

40. J Adv Nurs. Bias and prevalence effects on kappa viewed in terms of sensitivity and specificity. J Clin Epidemiol. 2000;53(5):499-503.

41. Fleiss JL, Cohen J. "The equivalence of weighted kappa and the intraclass correlation coefficient as measures of reliability" in Educational and Psychological Measurement. 1973; 33. p. 613-619

42. Millar E, Garland C, Ross F,et al. Practice nurses and the care of patients receiving depot neuroleptic treatment, views on training, confidence and use of structured assessment. J Adv Nurs. 1999;29(6):1454-1461.

43. Cleary A, Walsh F, Connolly H, et al. Monitoring and documentation of side effects from depot antipsychotic medication: an interdisciplinary audit of practice in a regional mental health service. $J$ Psychiatr Ment Health Nurs. 2012;19(5):395-401. 